'Servicio de Tumores Mamarios, Instituto Nacional de Cancerología, Ciudad de México, México.

22Área de Investigación, Instituto Nacional de Cancerología, Ciudad de México, México. ${ }^{3}$ Facultad de Medicina Mexicali, Universidad Autónoma de Baja California, Mexicali, Baja California, México.

${ }^{a}$ Residente Oncología Médica. besidente Ginecología Oncológica. cPhd. ${ }^{\mathrm{M}} \mathrm{MsC}$.

Los autores no refieren conflictos de intereses.

Fuente de apoyo financiero: No se recibieron apoyos para la realización de este trabajo.

Recibido el 11 de enero de 2017, aceptado el 19 de junio de 2017.

Correspondencia a: Jessica Elizabeth Salazar Campos Instituto Nacional de Cancerología, Ciudad de México, México.

Av. San Fernando No 22, Col. Sección XVI, Delegación Tlalpan, C.P. 14080, Ciudad de México, México.

jessica9salazar@hotmail.com

\section{Tumor Phyllodes con transformación a osteosarcoma}

\author{
TEREZA NIETO-CORONEL ${ }^{1, \mathrm{a}}$, JESSICA ELIZABETH SALAZAR-CAMPOS ${ }^{1, \mathrm{~b}}$, \\ DAVID CANTÚ DE LEÓN ${ }^{2, c}$, RAÚL DÍAZ-MOLINA ${ }^{3, c, d}$, \\ RAFAEL VÁZQUEZ-ROMO ${ }^{1, \mathrm{~d}}$, ENRIQUE BARGALLÓ-ROCHA ${ }^{1}$
}

\section{Breast osteosarcoma originating in a phyllodes tumor. Report of one case}

Phyllodes tumors account for less than $1 \%$ of tumors of the mammary gland, have both epithelial and stromal components and are classified as benign, borderline and malignant. The malignant tumors are highly heterogeneous: they can differentiate to liposarcomas, fibrosarcomas, rhabdomyosarcomas, chondrosarcomas or osteosarcomas. The differentiation to osteosarcoma is extremely rare, constitutes $1.3 \%$ of cases and is very aggressive. The standard treatment of these tumors is surgical. The role of radiotherapy and chemotherapy is not clear. However, in patients in whom wide surgical margins are not achieved, adjuvant radiotherapy can be of help. We report a 63 years old female with a right breast osteosarcoma with an osteoclastic component, originating in a phyllodes tumor. The tumor was excised surgically and afterwards she was treated with 10 sessions of $50 \mathrm{~Gy}$ of radiotherapy in 25 fractions. She has remained free of disease for the last 10 months.

(Rev Med Chile 2017; 145: 1076-1082)

Key words: Breast; Osteosarcoma; Phyllodes Tumor.
L os tumores phyllodes representan menos del $1 \%$ de los tumores mamarios ${ }^{1-3}$. Se caracterizan por presentar rápido crecimiento, se originan del estroma periductal y tienen componentes tanto epitelial como del estroma ${ }^{4}$. Se clasifican como benignos, borderline y malignos ${ }^{3,10,20}$, de acuerdo con la celularidad estromal, actividad mitótica, pleomorfismo nuclear y margen tumo$\mathrm{ral}^{10}$. Los tumores malignos son muy heterogéneos, pueden tener diferenciación a liposarcomas, fibrosarcomas, rabdomiosarcomas, condrosarcomas u osteosarcomas $^{5,7}$. La diferenciación a osteosarcoma es extremadamente rara ${ }^{2,3}$, constituye el 1,3\% de los casos y es altamente agresiva ${ }^{1,7}$. Con frecuencia esta diferenciación se presenta en grupos de edad media o edad adulta mayor, cursan asintomáticos, afectando el cuadrante superior externo en su mayoría, sin predominio de alguna glándula, con tamaños tumorales que varían desde 2 hasta
$40 \mathrm{~cm}^{3}$. Hasta el momento no existen factores de riesgo identificados ${ }^{6}$. Se reporta el caso de una paciente con tumor phyllodes maligno con diferenciación osteosarcomatosa de tipo osteoclástico.

\section{Presentación del caso}

Mujer de 63 años de edad, con hermana fallecida a los 50 años de edad por leiomiosacorma uterino, tía materna fallecida por cáncer de mama. Niega uso de terapia hormonal, menarquia a los 14 años, postmenopáusica. Inicia su cuadro con auto-detección de nódulo en mama derecha de seis meses de evolución. Al examen físico con tumor de $3,5 \times 3,5 \mathrm{~cm}$ en cuadrante superior interno en radio de 1 a 3 a $1 \mathrm{~cm}$ del pezón, sin afectar planos profundos ni piel, axila negativa. Mama y axila contralateral negativas. Se realizó mamografía con 
reporte de nódulo hiperdenso de bordes irregulares bien definidos, el cual se asocia a leve retracción del tejido adyacente [BIRADS 4 (Breast Imaging Reporting and Data System)], posteriormente se efectúa ultrasonido donde se observa en mama derecha en cuadrante superior interno en radio de 2 a 3 a $1 \mathrm{~cm}$ del pezón, un nódulo sólido de bordes lobulados parcialmente definidos, el cual muestra vascularidad interna a la aplicación del Doppler color, con medidas de 31,9 x 15,9 x 28,5 mm en sus diámetros mayores (Figura 1). Radiografía de tórax y ultrasonido abdominal negativos.

Se realizó biopsia trucut con reporte histopatológico de sarcoma de alto grado, con áreas de producción de material osteoide y células gigantes, compatible con osteosarcoma originado en tumor phyllodes (Figura 2 A, B y C). El estudio inmunohistoquímico arrojó los siguientes resultados: Receptor de estrógenos negativo [RE (-)], receptor de progesterona negativo $[R P(-)]$, receptor 2 del factor de crecimiento epidérmico humano negativo [HER2 (-)], marcador de proliferación celular Ki-67 15\%.

Se realizó escisión local amplia con reporte histopatológico definitivo compatible con os-

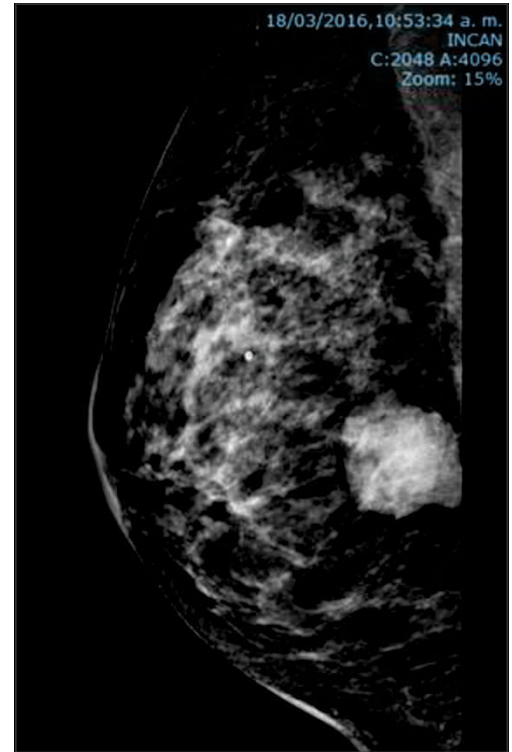

Figura 1. Glándula mamaria derecha: en cuadrante superior interno en radio de 2 a 3 a $1 \mathrm{~cm}$ de pezón, se observa nódulo sólido de bordes lobulados parcialmente definidos, el cual tiene vascularidad interna a la aplicación del Doppler color, con medidas de $31,9 \times 15,9 \times 28,5 \mathrm{~mm}$ en sus diámetros mayores. Presenta dilatación de un ducto en la región retroaereolar derecha en radio de 6 , de contenido anecoico.

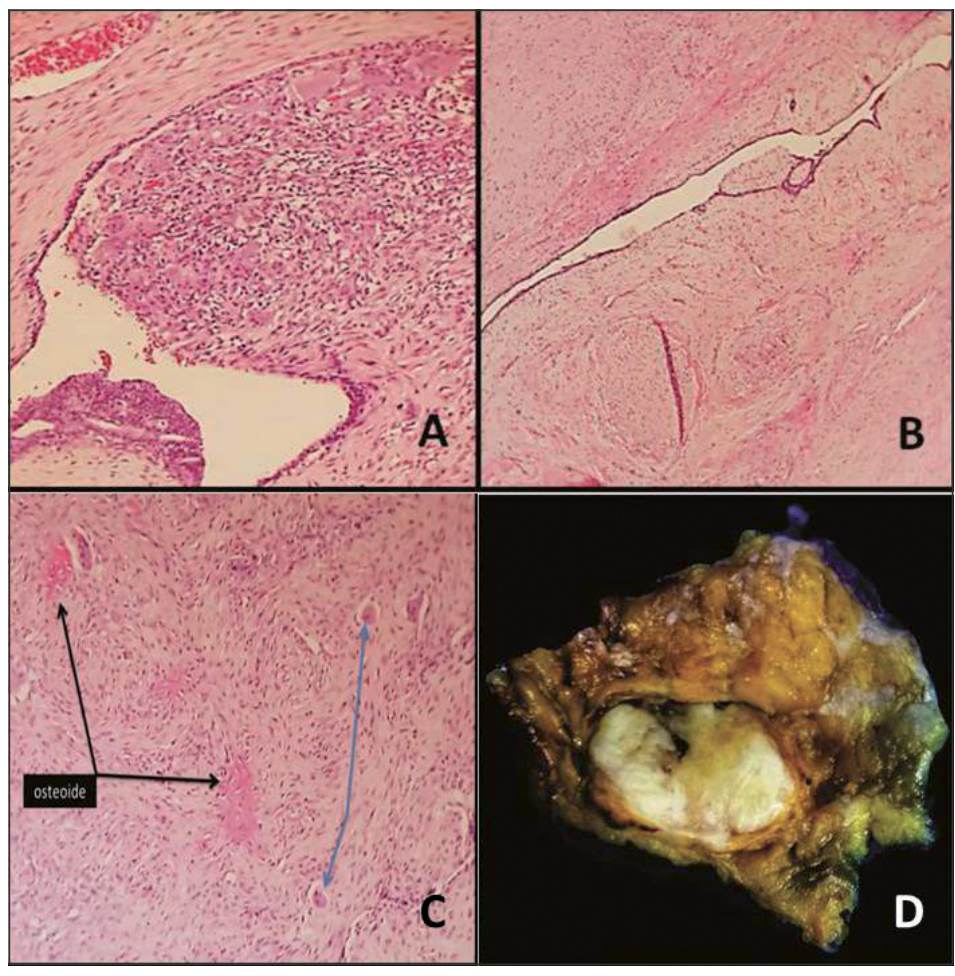

B

Figura 2. A) Se observa la presencia de una folea cubierta y sustituida por la neoplasia compuesta por osteoclastos y osteoblastos. B) Se identifican zonas donde aún persiste el tumor phyllodes de manera focal. C) Se observa la totalidad del parénquima mamario sustituido por la neoplasia maligna, con depósito de material amorfo, eosinófilo, que corresponde a osteoide. Las células gigantes multinucleadas corresponden a osteoclastos (flechas) y las células alargadas y mononucleares son osteoblastos. (Hematoxilina-eosina, 20x). D) Pieza macroscópica del tumor que al corte permite observar la lesión ovoide, mal definida, blanco perlada con áreas de aspecto mixoide, modular, granular y con hemorragia central focal. 
teosarcoma de células gigantes con componente osteoclástico, originado en tumor phyllodes, tamaño tumoral de $3,8 \times 2,8 \times 2,5 \mathrm{~cm}$, con bordes negativos. La pieza macroscópica al corte mostró una lesión ovoide, mal definida, blanco perlada con áreas de aspecto mixoide, nodular, granular y con hemorragia central focal (Figura 2D). Se indicó tratamiento adyuvante con radioterapia externa 50 Gy en 25 fracciones, actualmente con más de 10 meses de período libre de enfermedad.

\section{Discusión}

Se realizó una búsqueda intencionada en las bases de datos Pubmed/MEDLINE y Google Schoolar el 15 de septiembre de 2016, con las palabras clave "phyllodes and osteosarcomatous differentiation" y "phyllodes y diferenciación osteosarcomatoide". La búsqueda fue realizada por un médico residente del segundo grado de la subespecialidad en oncología médica y un médico especialista adscrito al Servicio de Tumores Mamarios del Instituto Nacional de Cancerología de México. Se seleccionaron las publicaciones que cumplieron con los siguientes criterios: publicaciones en inglés o español, casos con transformación maligna de tumor phyllodes a osteosarcoma sin otros componentes, que describan las características clínico-histopatológicas y/o terapéuticas de cada caso. La búsqueda arrojó 18 publicaciones de las cuales fueron excluidas tres: una por tratarse de un sarcoma tricoblástico con transformación a osteosarcoma, otra por tratarse de metaplasia con transformación ósea, y el tercero por tratarse de una diferenciación condrosarcomatosa. Las 15 publicaciones que cumplieron con los criterios de inclusión consideran un total de 36 casos de tumores phyllodes con transformación osteosarcomatosa. La Tabla 1 resume algunas características de estos 36 casos, así como del que nos ocupa en el presente trabajo.

Los tumores phyllodes malignos tienen alto riesgo de recurrencia y de desarrollar metástasis posterior al tratamiento escicional ${ }^{7}$, siendo los sitios metastásicos más frecuentes pulmón, hígado y hueso ${ }^{1}$, con una diseminación predominantemente por vía hematógena ${ }^{7}$. Los criterios para determinar la malignidad en estos tumores son hipercelularidad estromal, sobrecrecimiento estromal, células atípicas estromales, actividad mi- tótica alta, infiltración de los márgenes, así como la presencia de elementos heterólogos malignos ${ }^{2}$.

La transformación maligna de los tumores phyllodes se explica por diversas teorías, una de ellas sugiere una diferenciación de células embrionarias pluripotenciales, sin embargo, más del $40 \%$ de los sarcomas osteogénicos son originados de un fibroadenoma prexistente, lo que sugiere que esta neoplasia se origina de la metaplasia de células estromales que sufren transformación maligna ${ }^{1}$. El curso clínico de un sarcoma primario de mama y de un tumor phyllodes con transformación a osteosarcoma es similar ${ }^{3}$.

Silver et al. ${ }^{5}$ reportaron una serie de 22 casos de tumores phyllodes con diferenciación sarcomatosa donde el $73 \%$ presentó un tumor palpable, 4 pacientes se trataron con biopsia escisional, 6 con mastectomía simple, 10 con radical modificada y una con mastectomía radical. Las 11 pacientes sometidas a disección radical de axila, fueron todas negativas. El tamaño tumoral promedio fue de $6,4 \mathrm{~cm}$ (rango de 1,9 a $15 \mathrm{~cm}$ ), el componente osteosarcomatoso fue clasificado en fibroblástico, osteoclástico u osteoblástico, de los cuales se presentaron 11, 6 y 5 casos respectivamente. Con un seguimiento promedio de 44 meses, 9 pacientes desarrollaron recurrencia local, mientras que en 8 fue metastásica; 7 pacientes fallecieron dentro de los 12 meses de detección de la metástasis. Estos mismos autores observaron que el tamaño tumoral y el subtipo del componente osteosarcomatoso se correlacionó significativamente con el pronóstico de las pacientes, específicamente con la disminución del período libre de enfermedad en tumores de más de $5 \mathrm{~cm}$ y/o componentes osteoclástico u osteoblástico.

En lo que respecta al perfil molecular de estos tumores, hasta el $40 \%$ presentan receptores hormonales positivos, sin embargo, el tratamiento con hormonoterapia aún no está bien definido ${ }^{10}$. Es importante, al examinar el tejido tumoral, excluir las células epiteliales, ya que en el diagnóstico diferencial se debe considerar el carcinoma metaplásico con formación de hueso, el cual tiene un comportamiento y pronóstico totalmente diferente. Los tumores phyllodes con morfología benigna, pero que tienen un Ki-67 $>10 \%$ tienen riesgo de malignización ${ }^{8}$.El pronóstico es poco claro, sin embargo, se ha descrito la importancia de las siguientes características: índice de mitosis elevado, sobrecrecimiento estromal, pleomorfismo 


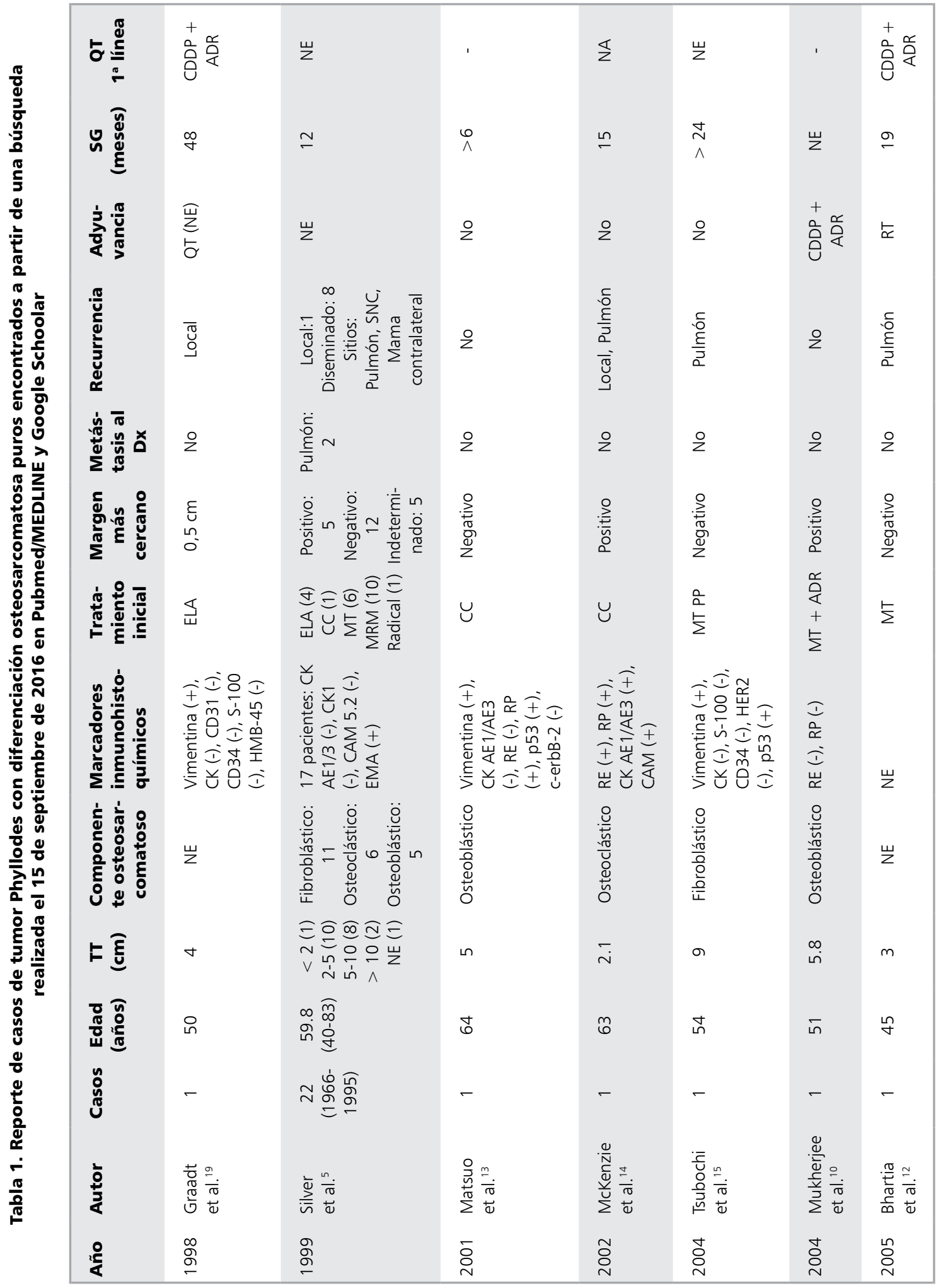




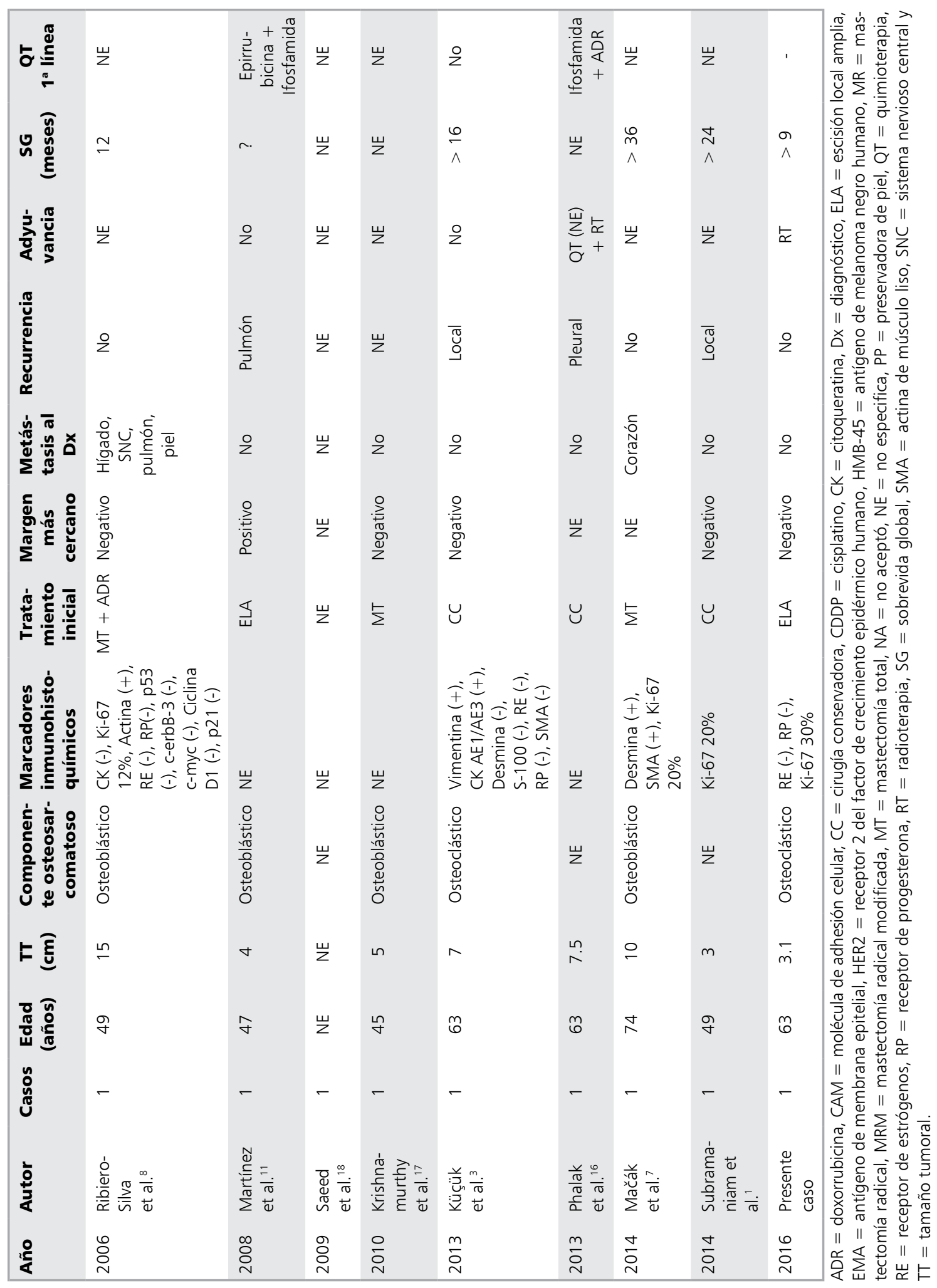


nuclear alto e infiltración de los márgenes ${ }^{9}$. Silver et al. ${ }^{5}$ documentaron que los tumores phyllodes con transformación a osteosarcoma pueden dar metástasis a distancia en 38\% de los casos, siendo más agresivos los osteoclásticos y osteoblásticos que los fibroblásticos; asimismo concluyeron que el componente osteosarcomatoso incrementa la mortalidad en 33\%. Las metástasis reportadas en la literatura principalmente son a pulmón, pleura, piel y hueso, pero también a otros sitios como corazón $^{7}$ e intestino ${ }^{2}$. El tratamiento estándar es quirúrgico, con la escisión completa del tumor con márgenes amplios o mastectomía total ${ }^{3}$. Sólo entre 10 y $15 \%$ de las pacientes pueden presentar adenopatías axilares palpables, que por lo general son secundarias a hiperplasia reactiva. La afectación tumoral axilar ocurre en menos de 5\% de las pacientes, por lo que la exploración quirúrgica axilar no se recomienda de forma rutinaria ${ }^{6,11}$.

Respecto a la adyuvancia no hay un consenso establecido, ya que se trata de un tumor extremadamente raro. En algunos casos se ha utilizado quimioterapia o radioterapia o ambas como tratamiento adyuvante. Algunos autores justifican el uso de radioterapia al no conseguir márgenes libres mayores de $1 \mathrm{~cm}$; por otro lado, se ha utilizado quimioterapia adyuvante, así como de rescate en recurrencias. Los esquemas utilizados de quimioterapia adyuvante o paliativa son ifosfamida más antraciclinas ${ }^{11} \mathrm{o}$ platino más antraciclinas ${ }^{12}$. En un reporte se documentó el uso de hasta tres líneas de tratamiento: ifosfamida más epirrubicina, metotrexate a dosis altas más vincristina, $y$ docetaxel más gemcitabina ${ }^{11}$.

\section{Conclusiones}

El tumor phyllodes con transformación osteosarcomatosa es extremadamente raro, su evolución es impredecible y son altamente agresivos. El tamaño tumoral y el componente osteosarcomatoso son los factores pronósticos más importantes. El tratamiento estándar principalmente es quirúrgico, el papel de la radioterapia y la quimioterapia aun no es claro, sin embargo, en pacientes que no se obtienen márgenes amplios mayores de $1 \mathrm{~cm}$ podrían ser llevados a radioterapia adyuvante. Los pacientes que presentan metástasis o recurrencia son candidatos a recibir quimioterapia paliativa, en la mayoría de los casos descritos se utilizaron esquemas con base en ifosfamida más antraciclinas (aunque en algunos casos se sustituyó la ifosfamida por platino). Por la rareza de este tipo de neoplasia el beneficio de la quimioterapia no está explorado y se extrapola de la evidencia en sarcoma de partes blandas y osteosarcomas, por lo que es indispensable realizar estudios prospectivos.

\section{Referencias}

1. Subramaniam J, Nair RP, Nayal B, Rao L. Malignant phyllodes with osteosarcomatous differentiation- A case report. Med Res Chron 2014; 1 (2): 193-6.

2. Al-Rabiy FN, Ali RH. Malignant phyllodes tumor with osteosarcomatous differentiation metastasizing to small bowel and causing intestinal obstruction. Diagn Histopathol 2014; 21 (4): 165-8.

3. Küçük Ü, Bayol Ü, Etit D, Gündüz F, Yücel S, Cumurcu $\mathrm{S}$, et al. Malignant phyllodes tumor of the breast with osteosarcomatous differentiation. J Breast Health 2013; 9 (2): 88-91.

4. Parker SJ, Harries SA, Phyllodes tumours. Postgrad Med J 2001; 77 (909): 428-35.

5. Silver SA, Tavassoli FA. Osteosarcomatous differentiation in phyllodes tumors. Am J Surg Pathol 1999; 23 (7): 815-21.

6. Guerrero MA, Ballard BR, Grau AM. Malignant phyllodes tumor of the breast: review of the literature and case report of stromal overgrowth. Surg Oncol 2003; 12 (1): 27-37.

7. Mačák J, Hurník P, Dvořáčková J, Mačáková J. An isolated metastasis to the heart from a malignant phyllodes tumor with osteosarcomatous differentiation. Cesk Patol 2014; 50 (4): 146-9.

8. Ribeiro-Silva A, Zambelli-Ramalho L, Zucoloto S. Phyllodes tumor with osteosarcomatous differentiation: a comparative immunohistochemical study between epithelial and mesenchymal cells. Tumori 2006; 92 (4): 340-6.

9. Hawkins RE, Schofield JB, Fisher C, Wiltshaw E, McKinna JA. The clinical and histologic criteria that predict metastases from cystosarcoma phyllodes. Cancer 1992; 69 (1): 141-7.

10. Mukherjee P, Kalish L, Delprado W, Crea P. Telangiectatic osteosarcomatous differentiation in a phyllodes tumour. ANZ J Surg 2004; 74 (8): 707-9.

11. Martínez A, Hardisson D, Muñoz M, Gómez F, de Santiago J. Transformación osteosarcomatosa de tumor phyllodes mamario. Rev Chil Obstet Ginecol 2008; 73 (4): 273-6. 
12. Bhartia SK, Kashyap P. Osteogenic pulmonary metastases originating from a phyllodes tumour of the breast with osteosarcomatous differentiation. Australas Radiol 2005; 49 (1): 63-5.

13. Matsuo K, Fukutomi T, Tsuda H, Hasegawa T, Akashi-Tanaka S, Nanasawa T. A Case of Malignant Phyllodes Tumor of the Breast with Osteosarcomatous Features. Breast Cancer 2001; 8 (1): 79-83.

14. McKenzie C, Philips J. Malignant phyllodes tumor metastatic to the lung with osteogenic differentiation diagnosed on fine needle aspiration biopsy. A case report. Acta Cytol 2002; 46 (4): 718-22.

15. Tsubochi H, Sato N, Kaimori M, Imai T. Osteosarcomatous differentiation in lung metastases from a malignant phyllodes tumour of the breast. J Clin Pathol 2004; 57 (4): 432-4.

16. Phalak K, Sedgwick EL, Dhamne S, Gutiérrez C. AIRP best cases in radiologic-pathologic correlation: malig- nant phyllodes tumor with osteosarcomatous differentiation. Radiographics 2013; 33 (5): 1377-81.

17. Krishnamurthy J. Osseous differentiation in cystosarcoma phyllodes-diagnosed by fine needle aspiration cytology. J Cytol 2010; 27 (4): 149-51.

18. Saeed SA, Masroor I. Malignant phyllodes tumour as an intracystic growth with osteosarcomatous differentiation. J Coll Physicians Surg Pak 2009; 19 (11): 723-5.

19. Graadt van Roggen J, Zonderland HM, Welvaart K, Peterse JL, Hogendoorn PC. Local recurrence of a phyllodes tumour of the breast presenting with widespread differentiation to a telangiectatic osteosarcoma. J Clin Pathol 1998; 51 (9): 706-8.

20. Aydogan F, Tasci Y, Sagara Y. Phyllodes Tumors of the Breast. In: Aydiner A, Igci A and Soran A (eds.), Breast Disease: Management and Therapies. Springer International Publishing Switzerland; 2016. p. 421-6. 